\title{
Sorting Out Fuzzy Transportation Problems via ECCT and Standard Deviation
}

\author{
Krishna Prabha Sikkannan, PSNA College of Engineering, India \\ (iD) https://orcid.org/0000-0002-5526-7701 \\ Vimala Shanmugavel, Mother Teresa Women's University, India
}

\begin{abstract}
A well-organized arithmetical procedure entitled standard deviation is employed to find the optimum solution in this paper. This technique has been divided into two parts. The first methodology deals with constructing the entire contingency cost table, and the second deals with optimum allocation. In this work, the method of magnitude is used for converting fuzzy numbers into crisp numbers as this method is better than the existing methods. This technique gives a better optimal solution than other methods. A numerical example for the new method is explained, and the authors compared their method with existing methods such as north west corner method, least cost method, and Vogel's approximation method.
\end{abstract}

\section{KEYWORDS}

Crisp Numbers, Defuzzification, Entire Contingency Cost Table, Method of Magnitude, Opportunity Cost, Standard Deviation

\section{INTRODUCTION}

The study of perfect transportation and distribution of measures in mathematics and economics is named as transportation theory and the presumption was dignified by Gaspard Monge (1781). The notable collapse on the charge and the pricing of raw materials and goods is visibly due to Transportation cost. An most favorable allotment sketch for a single commodity is established by the transportation problem. Our aim is to reduce entire carrying outlay for the possessions transferring from resource to target. By balancing the total supply and demand we can divide the transportation problem as balanced or unbalanced. Dual simplex, Big M method and Interior Approach method can also be used to unravel the transportation problem. Techniques like North West corner rule, row minima, column minima, matrix minima, or the vogel's approximation method are also applied to acquire the solution for transportation problems. Vogel's method provides fairly accurate elucidation whereas MODI and Stepping Stone (SS) techniques are measured as a customary procedure for achieving the best possible elucidation.

The cost of transportation is managed by dealer and manufacturer. Excluding the conventional methods like North West corner method, row minima method, least cost method, column minima method, Vogel's approximation method and modified distribution method etc, many researchers has endowed with new techniques to find a better initial basic feasible solution for the Transportation Problem. 
The entire constraints of the transportation problems may not be identified specifically owing to unmanageable issues in real world applications. The limitations of the transportation problem are not forever accurately documented and unwavering. This ambiguity may pursue from the need of literal data, faltering in decision etc. All the parameters of the transportation problems may not be known precisely due to uncontrollable factors in real world applications. The parameters of the transportation problem are not always precisely identified and sure. This imprecision may go after the lack of exact information, uncertainty in judgment etc. To overcome this situation Zadeh (1965) introduced the notion of fuzziness that was reinforced by Bellman and Zadeh (1965). Chanas and Kuchta (1996) proposed the concept of optimal solution for the transportation problem with fuzzy coefficients which are expressed as fuzzy numbers. A fuzzy number is thus a special case of a convex, normalized fuzzy set of the real line. Calculations with fuzzy numbers allow the incorporation of uncertainty on parameters, properties, geometry, initial conditions, etc. Membership functions (MFs) are the building blocks of fuzzy set theory, i.e., fuzziness in a fuzzy set is determined by its MF. Accordingly, the shapes of MFs are important for a particular problem since they effect on a fuzzy inference system. They may have different shapes like triangular, trapezoidal, Gaussian, etc. Since triangular membership function are fewer complexes when splitting values (low, med and high MF) comparing other membership functions. Considering that parameters of transportation problem have uncertainties, this paper develops a generalized fuzzy transportation problem with fuzzy supply, demand and cost. For simplicity, these parameters are assumed to be triangular fuzzy numbers in this paper.

Hajjari and Abbasbandy (2011) have proposed a new defuzzification method for fuzzy numbers. Mohanaselvi and Ganesan (2012) have found a fuzzy optimal solution to fuzzy transportation problem. Reena and Bhathawla $(2014,2016)$ provided an innovative and global approach to optimum solution of transportation problem. Pradeep et al. (2016) founded the solution of fuzzy transportation problem by using ranking functions. Urashikumari et al. (2017) applied the stepping stone method for solving the transportation problem. Krishna Prabha and Vimala (2016) applied BCM and Monalisha's Approximation Method for solving transportation problem. Palanivel and Suganya (2018) proposed a new method to solve transportation problem by using harmonic mean approach. Abul et al. (2017) have proposed average total opportunity cost method for solving transportation problems. Rizk-Allah et al. (2018) has presented a multi-objective transportation model under neutrosophic environment. Single valued neutrosophic hesitant fuzzy computational algorithm for multiobjective nonlinear optimization problem was proposed by Ahmad et al. (2018). Bi-level Linear Programming Problem with Neutrosophic Numbers was presented by Pramanik and Dey (2018). Exponential entropy for intuitionistic fuzzy sets and interval valued intuitionistic fuzzy sets was given by An-Peng Wei et al. (2019). Pythagorean approach to fuzzy transportation problems was proposed by Kumar and Edalatpanah (2019). Pentagonal Fuzzy Transportation Problem by ATM method was proposed by Charles and Chandrasekaran (2019). Optimal solution of balanced and unbalanced Fuzzy Transportation Problem by using octagonal fuzzy numbers was presented by Priyanka et al. (2016). Hari Ganesh et al. (2020) used distance based defuzzification method of various fuzzy quantities using centroid for solving the fuzzy transportation problem. Deng-Feng Li et al.(2020) used intervalvalued complex single-valued neutrosophic hesitant fuzzy generalized hybrid weighted averaging operators for decision making.

In spite of many existing methods for defuzzification like yagers ranking, centroid method, distance method etc, in this paper defuzzification of fuzzy numbers with method of magnitude has been introduced to get better accuracy. Further an Entire Contingency Cost Table is formulated and standard deviation is applied for solving the transportation problem which differs from other modes of finding the optimal solution. The computational process is easy and time complexity is reduced. The same problem is solved using various methods like north west corner, Matrix minima, Vogels approximation, row minima, column minima etc and the obtained results are compared with our proposed method. By applying this method we have obtained a best optimum solution. 
The Paper is divided into six sections, section one provides the introduction and section two offers the preliminaries, analysis of fuzzy transportation problem is provided in section three. Section four confers the algorithm for entire contingency cost table along with optimum allocation of Transportation Problem and section five furnishes the numerical examples, finally section six concludes the paper.

\section{PRELIMINARIES}

Definition 2.1: A fuzzy transportation Problem is assumed to be fuzzy balanced transportation problem if total supply is same as total demand.

Definition 2.2: A fuzzy transportation Problem is said to be fuzzy unbalanced transportation problem if total supply is not same as total demand.

Definition 2.3: A set of non-negative allocations $x_{i j}$ is said to be feasible solution of fuzzy linear programming problem if and only if it satisfies the row and column restrictions of the problem.

Definition 2.4: If there are $m$ equations in $(m+n)$ variables, then to solve these equations put any $n$ variables equal to zero and find the solution of $m$ equations in $m$ variables. If the obtained solution is unique, then it is termed as a basic solution, or else, it is termed as non-basic solution. The zero valued variables are called non-basic variables and the remaining variables are called basic variables.

Definition 2.5: The characteristic function $\mu_{A}$ of a crisp set $A \subseteq X$ assigns a value either 0 or 1 to each member in $X$.A function $\mu_{\bar{a}}$ such that the value assigned to the element of the universal set $X$ fall within a specified range i.e. $\mu_{\breve{a}}: \mathrm{X} \rightarrow[0,1]$. The assigned value indicates the membership grade of the element in the set $A$. The function $\mu_{\bar{a}(x)}$ is called the membership function and the set $\widehat{A}=\left\{\left(x, \quad \mu_{\widetilde{a}(x)} ; x \in X\right)\right\}$ defined by $\mu_{\widetilde{a}(x)}$ for each $x \in X$ is called a fuzzy set.

Definition 2.6: A fuzzy number ạ on $\mathrm{R}$ is said to be a triangular fuzzy number (TFN) or linear fuzzy number if its membership function ậ: $\mathrm{R} \rightarrow[0,1]$ has the following characteristics:

$\mu_{\hat{a}(x)}=\left\{\begin{array}{cc}\left(x-a_{1}\right) /\left(a_{2}-a_{1}\right) \text { if } a_{1} \leq x \leq a_{2} \\ \left(a_{3}-x\right) /\left(a_{3}-a_{2}\right) \text { if } a_{2} \leq x \leq a_{3} \\ 0 & \text { otherwise }\end{array}\right.$

We denote this triangular fuzzy number by ậ $=\left(a_{1}, a_{2}, a_{3}\right)$. We use $\mathrm{F}(\mathrm{R})$ to denote the set of all triangular fuzzy numbers.

Also if $\mathrm{m}=a_{2}$ represents the modal value or midpoint, $\alpha=\left(a_{2}-a_{1}\right)$ represents the left spread and $\beta=\left(a_{3}-a_{2}\right)$ represents the right spread of the triangular fuzzy number ậ $=\left(a_{1}, a_{2}, a_{3}\right)$, then the triangular fuzzy number ậ can be represented by the triplet ậ $=(\alpha, m, \beta)$ i.e. $(\alpha, m, \beta)$.

Defuzzification: Defuzzification is the process of finding singleton value (crisp value) which represents the average value of the TFNs. 


\subsection{Method of Magnitude}

Many different approaches for the ranking of fuzzy numbers have been proposed in the literature. Abbasbandy and Hajjari (2011) proposed a new ranking method based on the left and the right spreads at some $\alpha$-levels of fuzzy numbers.

For a triangular fuzzy number $\widetilde{a}=\left(a_{1}, a_{2}, a_{3}\right)=\left(a_{0}, a_{*}, a^{*}\right)$ with parametric for $\bar{a}=(\underline{a}(r), \bar{a}$ (r)) we define the magnitude of the triangular fuzzy number $\breve{a}$ by:

$\operatorname{Mag}(\breve{a})=\frac{1}{2}\left(\int_{0}^{1}\left(\bar{a}+\underline{a}+a_{0}\right) f(r) d r\right)=\frac{1}{2}\left(\int_{0}^{1}\left(a^{*}+4 a_{0}-a_{*}\right) f(r) d r\right)$

where the function $f(r)$ is a non-negative and increasing function on $[0,1]$ with $f(0)=0, f(1)=1$ and $\int_{0}^{1} f(r) d r=\frac{1}{2}$. The function $\mathrm{f}(\mathrm{r})$ can be considered as a weighting function. In real life applications, $\mathrm{f}(\mathrm{r})$ can be chosen by the decision maker according to the situation. In this paper, for convenience we use $f(r)=r$.

Hence:

$\operatorname{Mag}(\breve{a})=\left(\frac{a^{*}+4 a_{0}-a_{*}}{4}\right)=\left(\frac{\bar{a}+\underline{a}+a_{0}}{4}\right)$

The magnitude of a triangular fuzzy number $\breve{a}$ synthetically reflects the information on every membership degree, and meaning of this magnitude is visual and natural. Mag ( $\breve{a})$ is used to rank fuzzy numbers. The larger $\operatorname{Mag}(\vec{a})$, the larger fuzzy number.

For any two triangular fuzzy numbers $\breve{a}=\left(a_{0}, a_{*}, a^{*}\right)$ and $\breve{b}\left(b_{0}, b_{*}, b^{*}\right)$ :

1. $\operatorname{Mag}(\breve{a}) \geq \operatorname{Mag}(\breve{b})$ if and only if $\breve{a} \geq \breve{b}$

2. $\operatorname{Mag}(\breve{a}) \leq \operatorname{Mag}(\breve{b})$ if and only if $\breve{a} \leq \breve{b}$

3. $\operatorname{Mag}(\breve{a})=\operatorname{Mag}(\breve{b})$ if and only if $\breve{a} \approx \breve{b}$

\section{ANALYSIS OF FUZZY TRANSPORTATION PROBLEM}

Let $s_{i}$ indicates magnitude of artifact existing at source $i, d_{j}$ indicates magnitude of artifact needed at target $\mathrm{j}, \mathrm{C}_{\mathrm{ij}}$ indicates the cost of transporting one unit of artifact from source/origin $\mathrm{i}$ to target $\mathrm{j}$ and $\mathrm{x}_{\mathrm{ij}}$ indicates the magnitude transported from origin $\mathrm{i}$ to destination $\mathrm{j}$.

Assumptions: $\sum_{i=1}^{n} s_{i}=\sum_{j=1}^{m} d_{j}$. Here the transportation problem can be stated as a linear programming problem as:

minimize $z=\sum_{i=1}^{n} \sum_{j=1}^{m} c_{i j} * x_{i j}$

subject to: 
$\sum_{j=1}^{m} x_{i j}=s_{i}, \mathrm{i}=1,2,3 \ldots \mathrm{n}$

$\sum_{i=1}^{n} x_{i j} d_{j}, \mathrm{j}=1,2,3 \ldots \mathrm{m}$

and:

$x_{i j} \geq 0$ for all $i=1,2, \ldots n$, and $j=1,2 \ldots m$

Clearly, for any of the parameters $\mathrm{c}_{\mathrm{ij}}$, $s_{i}$, or $d_{j}$ is fuzzy, the total transportation cost $\mathrm{z}$ is fuzzy as well. Here, $\mu_{\tilde{c}_{i j}}, \mu_{\tilde{s}_{i}}$, and $\mu_{\tilde{d}_{j}}$ denote their membership functions.

$\mathrm{s}\left(\tilde{c}_{i j}\right), \mathrm{s}\left(\tilde{s}_{i}\right)$, and $\mathrm{s}\left(\tilde{d}_{j}\right)$ are the supports of $\tilde{c}_{i j}, \tilde{s}_{i}$ and $\tilde{d}_{j}$ which represent the universal sets of the unit shipping cost, the quantity supplied by $\mathrm{i}^{\text {th }}$ supplier and the quantity required by $\mathrm{j}^{\text {th }}$ customer, respectively.

The fuzzy transportation problem is given by:

$\operatorname{minimize} \tilde{z}=\sum_{\mathrm{i}=1}^{\mathrm{n}} \sum_{\mathrm{j}=1}^{\mathrm{m}} \tilde{c}_{i j}{ }^{*} \mathrm{x}_{\mathrm{ij}}$

subject to:

$\sum_{j=1}^{m} x_{i j}=\tilde{s}_{i}, \mathrm{i}=1,2,3 \ldots \mathrm{n}$

$\sum_{i=1}^{n} x_{i j}, \tilde{d}_{j}, \mathrm{j}=1,2,3 \ldots \mathrm{m}$

and:

$\mathrm{x}_{\mathrm{ij}} \geq 0$, for all $\mathrm{i}=1,2, \ldots \mathrm{n}$, and $\mathrm{j}=1,2, \ldots \mathrm{m}$

All the demands, supplies are considered as fuzzy numbers. Crisp costs can be symbolized by degenerated membership functions in which, only one value is in their domains.

\section{MODIFIED ALGORITHM}

The developed algorithm in the current research involves two parts:

1. Algorithm for Entire Contingency Cost Table (ECCT);

2. Algorithm for optimum allocation of Transportation Problem. 
Table 1. Fuzzy Transportation Problem

\begin{tabular}{|c|c|c|c|c|c|}
\hline Origin(i) & $\mathbf{1}$ & $\mathbf{2}$ & $\mathbf{3}$ & & Supply(s) \\
\hline 1 & $\mathrm{X}_{11}$ & $\mathrm{X}_{12}$ & $\mathrm{X}_{13}$ & $\mathrm{X}_{1 \mathrm{n}}$ & $\mathrm{s}_{1}$ \\
\hline 2 & $\mathrm{C}_{11}$ & $\mathrm{C}_{12}$ & $\mathrm{C}_{13}$ & $\mathrm{C}_{1 \mathrm{n}}$ & $\mathrm{s}_{2}$ \\
\hline- & $\mathrm{X}_{21}$ & $\mathrm{X}_{22}$ & $\mathrm{X}_{23}$ & $\mathrm{X}_{2 \mathrm{n}}$ & \\
\hline- & $\mathrm{C}_{21}$ & $\mathrm{C}_{22}$ & $\mathrm{C}_{23}$ & $\mathrm{C}_{2 \mathrm{n}}$ & \\
\hline$M$ & - & - & - & - & \\
\hline Demand(dj) & & & & & $\mathrm{S}_{\mathrm{n}}$ \\
\hline
\end{tabular}

\subsection{Algorithm for ECCT}

Step 1: From the given TT, deduct the least value from each of the elements of every row and consign them on the right-top of subsequent elements.

Step 2: In each column deduct the least value from each element and place them on the right-foot of the corresponding elements.

Step 3: Add the right-top and right-foot elements of Steps 1 and 2 and frame the ECCT.

\subsection{Algorithm for Allocation}

Step 1: Calculate the standard deviation of Entire Contingency costs of cells along each row known as Row Standard Deviation Total Opportunity Cost (RSDTOC) and the standard deviation of Entire Contingency costs of cells along each column known as Column Standard Deviation Total Opportunity Cost (CSDTOC) just subsequent to and beneath the supply and demand amount correspondingly inside the first brackets.

Step 2: Spot the largest element among the RSDTOCs and CSDTOCs, in case of tie i.e, if there is more than one largest element then select the largest element along which the smallest cost element is present. If there is more than one smallest element, select any one of them arbitrarily.

Step 3: Allocate $x_{i j}=\min \left(a_{i}, b_{j}\right)$ on the left top of the least entry in the $(\mathrm{i}, \mathrm{j})^{\text {th }}$ of the TT.

Step 4: See below:

- If $a_{i}<b_{j}$, leave the $\mathrm{i}^{\text {th }}$ row and find $b_{j}^{!}=b_{j}-a_{i}$;

- If $a_{i}>b_{j}$, leave the $\mathrm{j}^{\text {th }}$ column and find $b_{j}^{!}=a_{i}-b_{j}$;

- If $a_{i}=b_{j}$, leave either $\mathbf{i}^{\text {th }}$ row or $\mathbf{j}^{\text {th }}$ column but not both.

Step 5: Redo the Steps 1 to 4 until the rim condition is attained.

Step 6: Evaluate, $\mathrm{Z}=\sum_{i=1}^{m} \sum_{j=1}^{n} C_{i j} X_{i j}$, where $\mathrm{Z}$ is the minimum transportation cost, $C_{i j}$ is the cost element of the TT.

\section{NUMERICAL EXAMPLE}

\subsection{Example 1}

Consider the following Transportation Problem. A firm has four factories O1, O2, O3 and O4 that fabricate machine components in four different places. The firm manager would like to transport the machine components from factories to four different warehouses D1, D2, D3 and D4. 
Table 2. Triangular Fuzzy Transportation Problem

\begin{tabular}{|c|c|c|c|c|c|}
\hline & D1 & D2 & D3 & D4 & SUPPLY \\
\hline O1 & $(2,3,4)$ & $(6,7,8)$ & $(4,5,6)$ & $(4,5,6)$ & $(37,38,39)$ \\
\hline O2 & $(4,5,6)$ & $(4,5,6)$ & $(2,3,4)$ & $(3,4,5)$ & $(18,19,20)$ \\
\hline O3 & $(1,2,3)$ & $(3,4,5)$ & $(4,5,6)$ & $(4,5,6)$ & $(15,16,17)$ \\
\hline O4 & $(3,4,5)$ & $(8,9,10)$ & $(3,4,5)$ & $(5,6,7)$ & $(22,23,24)$ \\
\hline DEMAND & $(24,25,26)$ & $(29,30,31)$ & $(20,21,22)$ & $(20,21,22)$ & \\
\hline
\end{tabular}

Converting the triangular fuzzy numbers into crisp numbers using the formula:

$\operatorname{Mag}(\breve{a})=\left(\frac{a^{*}+4 a_{0}-a_{*}}{4}\right)=\left(\frac{\bar{a}+\underline{a}+a_{0}}{4}\right)$
$R_{11}(2,3,4)=\frac{4+4^{*} 2-3}{4}=\frac{4+8-3}{4}=\frac{9}{4}=2.25$

similarly proceed for all fuzzy numbers.

Since the total supply is equal to total demand:

$\sum_{j=1}^{m} x_{i j}=\tilde{s}_{i}, 93=\sum_{i=1}^{n} x_{i j}, \tilde{d}_{j}$

the given problem is a balanced problem.

\subsection{Formation of Entire Contingency Cost Table (ECCT)}

From the given TT, deduct the least value from each of the elements of every row and consign them on the right-top of subsequent elements. In each column deduct the least value from each element and place them on the right-foot of the corresponding elements. Add the right-top and right-foot elements of Steps 1 and 2 and frame the ECCT.

\subsection{Allocation of the Cost With Supply and Demand}

Step 1: Calculate the standard deviation of Entire Contingency costs of cells along each row and each column just subsequent to and beneath the supply and demand amount correspondingly inside the first brackets.

Table 3. Crisp Transportation Problem

\begin{tabular}{|l|c|c|c|c|c|}
\hline & D1 & D2 & D3 & D4 & SUPPLY \\
\hline O1 & 2.25 & 6.25 & 4.25 & 4.25 & 37.25 \\
\hline O2 & 4.25 & 4.25 & 2.25 & 3.25 & 18.25 \\
\hline O3 & 1.25 & 3.25 & 4.25 & 4.25 & 15.25 \\
\hline O4 & 3.25 & 8.25 & 3.25 & 5.25 & 22.25 \\
\hline & 24.25 & 28.25 & 20.25 & 20.25 & 93 \\
\hline
\end{tabular}


Table 4. Entire Contingency Cost

\begin{tabular}{|l|c|c|c|c|c|}
\hline & D1 & D2 & D3 & D4 & SUPPLY \\
\hline O1 & 1 & 7 & 4 & 3 & 37.25 \\
\hline O2 & 5 & 3 & 0 & 1 & 18.25 \\
\hline O3 & 0 & 2 & 5 & 4 & 15.25 \\
\hline O4 & 2 & 5 & 1 & 4 & 22.25 \\
\hline & 24.25 & 28.25 & 20.25 & 20.25 & \\
\hline
\end{tabular}

Table 5. Row /Column Standard Deviation Total Opportunity Cost

\begin{tabular}{|c|c|c|c|c|c|c|}
\hline & D1 & D2 & D3 & D4 & SUPPLY & \\
\hline O1 & 1 & 7 & 4 & 3 & 37.25 & {$[2.16]$} \\
\hline O2 & 5 & 3 & 0 & 1 & 18.25 & {$[1.92]$} \\
\hline O3 & 0 & 2 & 5 & 4 & 15.25 & {$[1.92]$} \\
\hline O4 & 2 & 5 & 1 & 4 & 22.25 & {$[1.58]$} \\
\hline DEMAND & 24.25 & 28.25 & 20.25 & 20.25 & & \\
\hline
\end{tabular}

Step 2: Spot the largest element among the RSDTOCs and CSDTOCs, in case of tie i.e, if there is more than one largest element then select the largest element along which the smallest cost element is present.

Here the maximum value 2.16 is along the first row. Select the least cost in that row and allocate the values and balance the table. If there is more than one smallest element, select any one of them arbitrarily.

Delete the first column and find the standard deviation for each and every row and column. Select the maximum standard deviation. The maximum value is along the second column.

Step 3: Allocate $x_{i j} \min \left(a_{i}, b_{j}\right)$ on the left top of the least entry in the $(\mathrm{i}, \mathrm{j})^{\text {th }}$ of the TT.

Step 4: See below:

Table 6. R/C SD Total Opportunity Cost-1

\begin{tabular}{|c|c|c|c|c|c|c|}
\hline & D1 & D2 & D3 & D4 & SUPPLY & \\
\hline O1 & $1(24.25)$ & 7 & 4 & 3 & $37.25(13)$ & {$[2.16]$} \\
MAX \\
\hline O2 & 5 & 3 & 0 & 1 & 18.25 & {$[1.92]$} \\
\hline $\mathbf{O 3}$ & 0 & 2 & 5 & 4 & 15.25 & {$[1.92]$} \\
\hline $\mathbf{O 4}$ & 2 & 5 & 1 & 4 & 22.25 & {$[1.58]$} \\
\hline DEMAND & $24.25(0)$ & 28.25 & 20.25 & 20.25 & & \\
\hline & {$[1.87]$} & {$[1.92]$} & {$[2.06]$} & {$[1.22]$} & & \\
\hline
\end{tabular}


Table 7. R/C SD Total Opportunity Cost

\begin{tabular}{|c|c|c|c|c|c|c|c|c|c|c|}
\hline & D1 & D2 & D3 & D4 & SUPPLY & & & & & \\
\hline 01 & $\begin{array}{c}1 \\
(24.25) \\
\end{array}$ & 7 & 4 & $\begin{array}{c}3 \\
(13) \\
\end{array}$ & 37.25 & $\begin{array}{l}{[2.16]} \\
\text { MAX }\end{array}$ & [1.69] & [1.69] & $\begin{array}{l}{[1.69]} \\
\text { MAX }\end{array}$ & \\
\hline 02 & 5 & 3 & $\begin{array}{c}0 \\
(18.25) \\
\end{array}$ & 1 & 18.25 & {$[1.92]$} & {$[1.24]$} & & & \\
\hline 03 & $\begin{array}{c}0 \\
(15.25) \\
\end{array}$ & 2 & 5 & 4 & 15.25 & {$[1.92]$} & {$[1.24]$} & {$[1.24]$} & & \\
\hline 04 & 2 & $\begin{array}{c}5 \\
(13) \\
\end{array}$ & $\begin{array}{c}1 \\
(2)\end{array}$ & $\begin{array}{c}4 \\
(7.25) \\
\end{array}$ & 22.25 & [1.58] & [1.69] & [1.69] & [1.69] & [1.69] \\
\hline \multirow[t]{5}{*}{ DEMAND } & 24.25 & 28.25 & 20.25 & 20.25 & & & & & & \\
\hline & {$[1.87]$} & [1.92] & {$[2.06]$} & {$[1.22]$} & & & & & & \\
\hline & & [1.92] & $\begin{array}{l}{[2.06]} \\
\text { MAX }\end{array}$ & {$[1.22]$} & & & & & & \\
\hline & & $\begin{array}{l}{[2.05]} \\
\text { MAX }\end{array}$ & [1.69] & {$[0.47]$} & & & & & & \\
\hline & & [1] & {$[1.5]$} & {$[0.5]$} & & & & & & \\
\hline
\end{tabular}

- If $a_{i}<b_{j}$, leave the $\mathrm{i}^{\text {th }}$ row and find $b_{j}^{!}=b_{j}-a_{i}$;

- If $a_{i}>b_{j}$, leave the $\mathrm{j}^{\text {th }}$ column and find $b_{j}^{!}=a_{i}-b_{j}$;

- If $a_{i}=b_{j}$, leave either $\mathrm{i}^{\text {th }}$ row or $\mathrm{j}^{\text {th }}$ column but not both.

After making the required allocations we can frame the final optimum table by considering the above values.

The optimum cost is given by:

$(2.25 \times 24.25)+(4.25 \times 13)+(1.25 \times 15.25)+(2.25 \times 18.25)+(8.25 \times 13)+(5.25 \times 7.25)+(3.25 \times 2)$

$=54.56+55.25+19.06+41.06+107.25+38.06+6.5=302.8$

By solving the same problem with various methods like north west corner, least cost and vogels approximation method we get the following results.

\subsection{Allocation by North West Corner Rule}

See Table 9.

The optimum cost is given by:

$(2.25 \times 24.25)+(6.25 \times 13)+(4.25 \times 15.25)+(2.25 \times 3)+(4.25 \times 15.25)+(3.25 \times 2.25)$

$+(5.25 \times 20.25)$

$=54.56+81.25+64.81+6.75+64.81+7.31+106.31=385.84$

Table 8. EECT Total Opportunity Cost

\begin{tabular}{|c|c|c|c|c|}
\hline & D1 & D2 & D3 & D4 \\
\hline O1 & $2.25(24.25)$ & 6.25 & $4.25(13)$ & 4.25 \\
\hline $\mathbf{O 2}$ & 4.25 & 4.25 & $2.25(18.25)$ & 3.25 \\
\hline $\mathbf{O 3}$ & $1.25(15.25)$ & 3.25 & 4.25 & 4.25 \\
\hline $\mathbf{O 4}$ & 3.25 & $8.25(13)$ & $3.25(2)$ & $5.25(7.25)$ \\
\hline
\end{tabular}


Table 9. North West Corner Allocation-Final

\begin{tabular}{|l|c|c|c|c|}
\hline & D1 & D2 & D3 & D4 \\
\hline O1 & $2.25(24.25)$ & $6.25(13)$ & 4.25 & 4.25 \\
\hline O2 & $4.25(15.25)$ & 4.25 & $2.25(3)$ & 3.25 \\
\hline O3 & 1.25 & 3.25 & $4.25(15.25)$ & 4.25 \\
\hline O4 & 3.25 & 8.25 & $3.25(2.25)$ & $5.25(20.25)$ \\
\hline
\end{tabular}

Table 10. Matrix minima method-Final

\begin{tabular}{|c|c|c|c|c|}
\hline & D1 & D2 & D3 & D4 \\
\hline O1 & $2.25(9)$ & $6.25(8.25)$ & 4.25 & $4.25(20.25)$ \\
\hline O2 & 4.25 & 4.25 & $2.25(18.25)$ & 3.25 \\
\hline O3 & $1.25(15.25)$ & 3.25 & 4.25 & 4.25 \\
\hline O4 & 3.25 & $8.25(20.25)$ & $3.25(2)$ & 5.25 \\
\hline
\end{tabular}

\subsection{Matrix Minima Method}

See Table 10.

The optimum cost is given by:

$(2.25 \times 9)+(6.25 \times 8.25)+(4.25 \times 20.25)+(2.25 \times 18.25)+(1.25 \times 15.25)$

$+(8.25 \times 20.25)+(3.25 \times 2)$

$=20.25+51.56+86.06+41.06+19.06+167.06+6.5=391.5$

\subsection{Vogel's Approximation Method}

See Tables 11 and 12.

The optimum cost is given by:

$(2.25 \times 24.25)+(4.25 \times 13)+(4.25 \times 13)+(3.25 \times 15.25)+(3.25 \times 15.25)+(3.25 \times 20.25)+(5.25 \times 2)$ $=54.56+55.25+55.25+49.56+17.06+65.81+10.5=307.68$

\subsection{Example 2}

See Table 13.

Table 11. Vogel's Approximation method-Final

\begin{tabular}{|c|c|c|c|c|}
\hline & D1 & D2 & D3 & D4 \\
\hline O1 & $2.25(24.25)$ & 6.25 & $4.25(13)$ & 4.25 \\
\hline O2 & 4.25 & $4.25(13)$ & 2.25 & $3.25(5.25)$ \\
\hline O3 & 1.25 & $3.25(15.25)$ & 4.25 & 4.25 \\
\hline O4 & 3.25 & 8.25 & $3.25(20.25)$ & $5.25(2)$ \\
\hline
\end{tabular}


Table 12. Comparison of Methods

\begin{tabular}{|c|c|c|c|c|c|}
\hline Column Minima & Row Minima & NWC & MMM & VAM & Proposed Method \\
\hline 333.25 & 367.25 & 385.83 & 391.55 & 307.68 & 302.87 \\
\hline
\end{tabular}

Figure 1. Example 1: Comparison chart

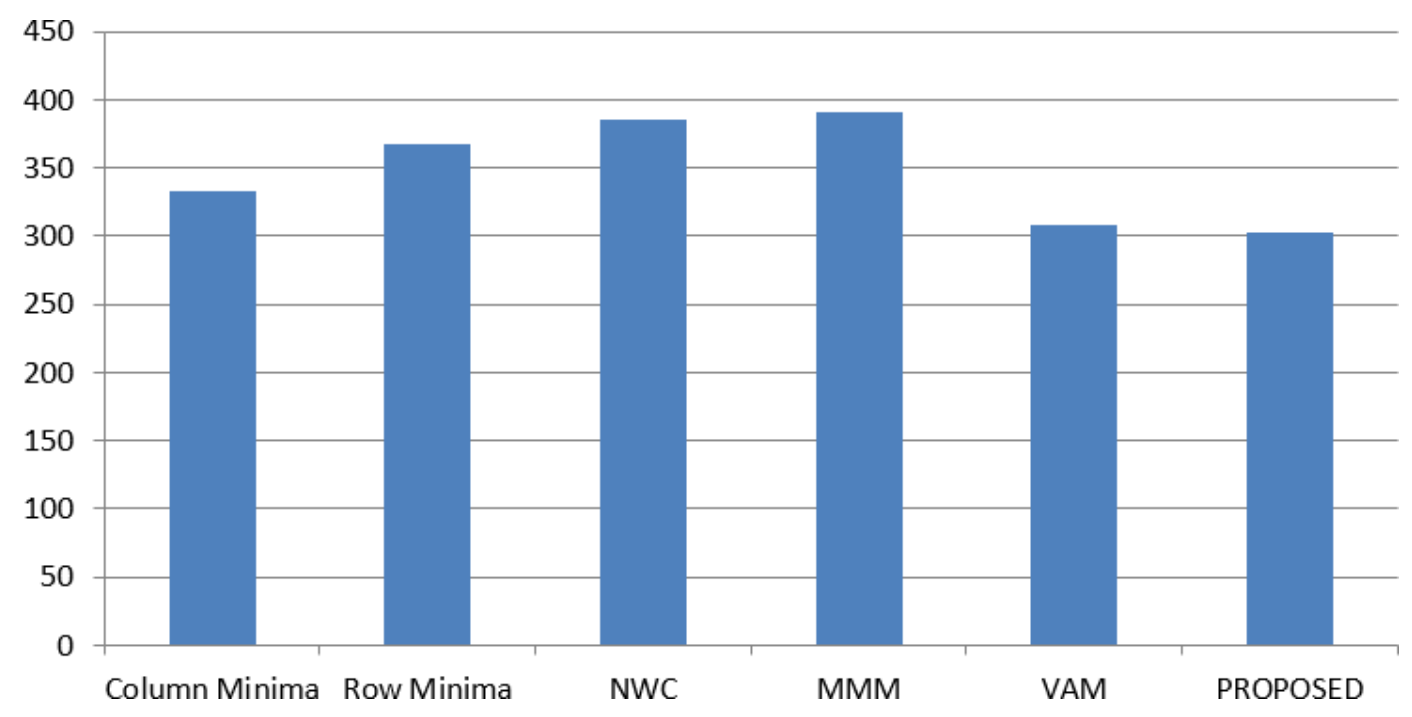

\subsection{Deffuzzification by Method of Magnitude}

$R_{11}(1,3,5)$

$=\frac{5+4^{*} 1-3}{4}=\frac{6}{4}=1.5$

See Tables 14-17.

The optimum cost is given by:

$1.5 \times 7.5+3.5 \times 3+2.5 \times 3.5+4.5 \times 5.5+5.5 \times 3$

$11.25+10.5+8.75+24.75+16.5=71.75$

Table 13. Triangular Fuzzy Transportation Problem

\begin{tabular}{|l|c|c|c|c|}
\hline & D1 & D2 & D3 & \\
\hline S1 & $(1,3,5)$ & $(1,3,5)$ & $(3,5,7)$ & $(7,9,11)$ \\
\hline S2 & $(4,6,8)$ & $(3,5,7)$ & $(2,4,6)$ & $(6,8,10)$ \\
\hline S3 & $(4,6,8)$ & $(8,10,12)$ & $(5,7,9)$ & $(8,10,12)$ \\
\hline & $(5,7,9)$ & $(10,12,14)$ & $(6,8,10)$ & \\
\hline
\end{tabular}


Table 14. Crisp Transportation Problem

\begin{tabular}{|l|l|l|l|l|}
\hline & D1 & D2 & D3 & \\
\hline S1 & 1.5 & 1.5 & 3.5 & 7.5 \\
\hline S2 & 4.5 & 3.5 & 2.5 & 6.5 \\
\hline S3 & 4.5 & 8.5 & 5.5 & 8.5 \\
\hline & 5.5 & 10.5 & 6.5 & \\
\hline
\end{tabular}

Table 15. Entire Contingency Cost

\begin{tabular}{|c|c|c|c|c|}
\hline & D1 & D2 & D3 & \\
\hline S1 & 0 & 0 & 3 & 7.5 \\
\hline S2 & 5 & 3 & 0 & 6.5 \\
\hline S3 & 3 & 11 & 4 & 8.5 \\
\hline & 5.5 & 10.5 & 6.5 & \\
\hline
\end{tabular}

Table 16. EECT Total Opportunity

\begin{tabular}{|l|c|c|c|c|}
\hline & D1 & D2 & D3 & \\
\hline S1 & 1.5 & $1.5,7.5$ & 3.5 & 7.5 \\
\hline S2 & 4.5 & $3.5,3$ & $2.5,3.5$ & 6.5 \\
\hline S3 & $4.5,5.5$ & 8.5 & $5.5,3$ & 8.5 \\
\hline & 5.5 & 10.5 & 6.5 & \\
\hline
\end{tabular}

Table 17. Comparison Cost

\begin{tabular}{|c|c|c|c|c|c|}
\hline Column Minima & Row Minima & NWC & MMM & VAM & Proposed Method \\
\hline 86.75 & 77.75 & 86.75 & 99.75 & 86.75 & 71.75 \\
\hline
\end{tabular}

\section{CONCLUSION}

The method of magnitude ranking is applied for defuzzifying the fuzzy problem which is different from other methods. An Entire Contingency Cost Table (ECCT) has been created by finding Standard Deviation for each row and column and the optimum allocation is made. By comparing with the existing methods like North West Corner, Matrix Minima Method and Vogel's Approximation Method, the proposed method gives a better approximation. This method is more optimum than other methods and provides a better optimum solution. This technique can be applied in various types of problems in assignment, transshipment etc.This method can be tested for trapezoidal, nanoganal and pentagonal fuzzy numbers etc.Real time problems can be analyzed by taking samples and applying this method. 
Figure 2. Example 2: Comparison chart

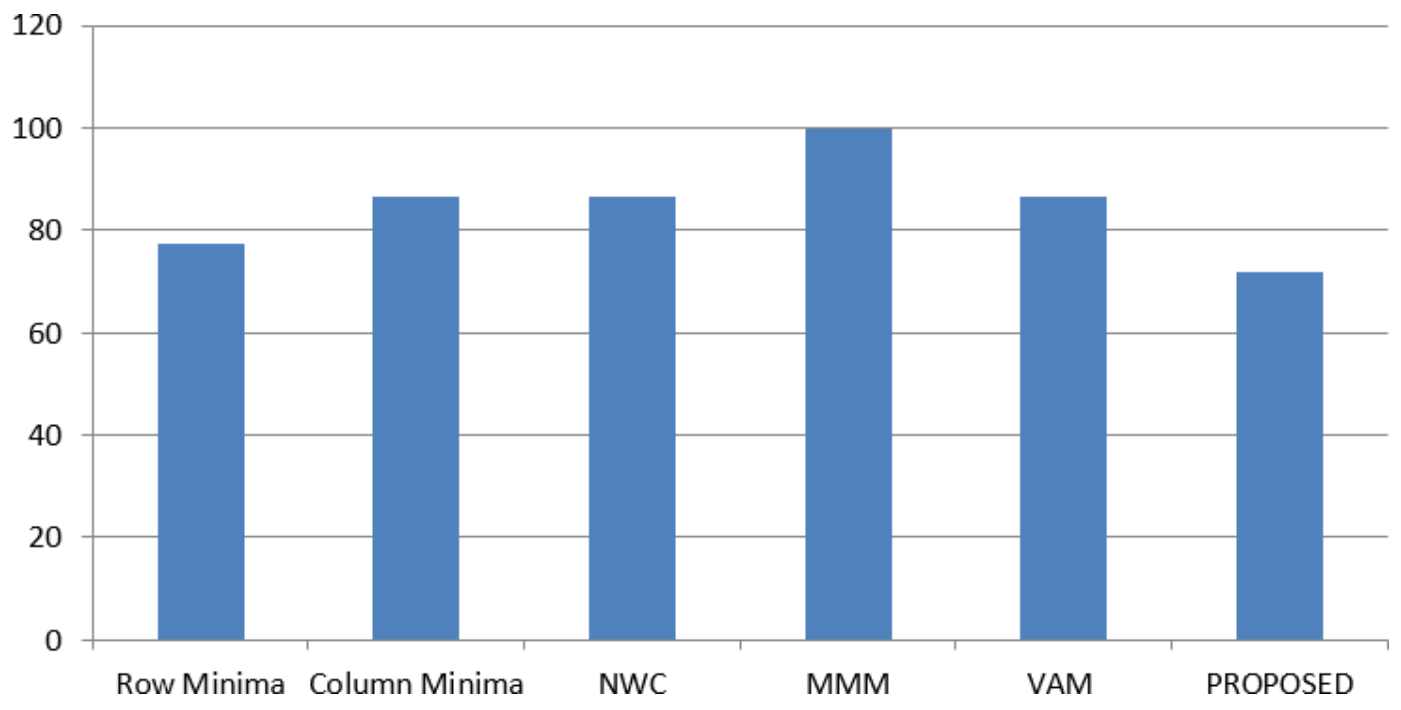




\section{REFERENCES}

Abul Kalam Azad, S. M., Bellel Hossain, Md., \& Mizanur Rahman, Md. (2017). An algorithmic approach to solve transportation problems with the average total opportunity cost method. International Journal of Scientific and Research Publications, 7(2).

Ahmad, F., Adhami, A. Y., \& Smarandache, F. (2018). Single valued neutrosophic hesitant fuzzy computational algorithm for multiobjective nonlinear optimization problem. Infinite Study.

Chanas, S., \& Kuchta, D. (1996). A concept of the optimal solution of the transportation problem with fuzzy cost coefficients. Fuzzy Sets and Systems, 82, 299-305.

Charles Rabinson, G., \& Chandrasekaran, R. (2019). Method for Solving a Pentagonal Fuzzy Transportation Problem via Ranking Technique and ATM. International Journal of Research in Engineering. IT and Social Sciences, 9(4), 71-75.

Ganesh, Suresh, \& Sivakumar. (2020). On solving fuzzy transportation problem based on distance based defuzzification method of various fuzzy quantities using centroid. Malaya Journal of Matematik, S(1), 410-426.

Hajjari, T., \& Abbasbandy, S. (2011). A Promoter Operator for Defuzzification Methods. Australian Journal of Basic and Applied Sciences, 5(10), 1096-1105.

Krishna Prabha, S., \& Vimala, S. (2016). Implementation of BCM for Solving the Fuzzy Assignment Problem with Various Ranking Techniques. Asian Research Journal of Mathematics, 1(2), 1-11.

Kumar, R., \& Edalatpanah, S. A. (2019). A Pythagorean fuzzy approach to the transportation problem. Complex \& Intelligent Systems, 5, 255-263.

Li, , Mahmood, Ali, \& Dong. (2020). Decision making based on interval-valued complex single-valued neutrosophic hesitant fuzzy generalized hybrid weighted averaging operators. Journal of Intelligent \& Fuzzy Systems, 38(4), 4359-4401.

Pramanik, S., \& Dey, P. P. (2018). Bi-level Linear Programming Problem with Neutrosophic Numbers. Neutrosophic Sets \& Systems, 21.

Priyanka, , Pathade, \& Ghadle. (2016). Optimal Solution of Balanced and Unbalanced Fuzzy Transportation Problem By Using Octagonal Fuzzy Numbers. International Journal of Pure and Applied Mathematics, 119(4), $617-625$.

Reena, P. G., \& Bhathawla, P. H. (2016). An Innovative Approach to Optimum Solution of Transportation Problem. International Journal of Innovative Research in Science. Engineering Technology, 5(4), 5695-5700.

Reena, P. G., \& Bhathawla, P. H. (2014). The New Global Approach to Transportation Problem. International Journal of Engineering Technology. Management and Applied Science, 2(3), 109-113.

Rizk-Allah, R. M., Hassanien, A. E., \& Elhoseny, M. (2018). A multi-objective transportation model under neutrosophic environment. Computers \& Electrical Engineering, 69, 705-719.

Sahoo, Behera, \& Jyoti. (2016). Solution of Fuzzy Transportation Problem by Using Ranking Function. Advanced Science Letters, 22(2), 564-566.

Urashikumari, P. D., Dhavakumar, R., \& Bhasvar, C. (2017). Transportation Problem Using Stepping Stone Method and its Application. International Journal of Advanced Research in Electrical, electronics and Instrumentation Engineering, 6(1), 46-50.

Vimala, S., \& Krishna Prabha, S. (2016). Fuzzy Transportation Problem through Monalisha's Approximation Method. British Journal of Mathematics \& Computer Science, 17(2), 1-11.

Wei, A.-P., Li, D.-F., Jiang, B.-Q., \& Lin, P.-P. (2019). The novel generalized exponential entropy for intuitionistic fuzzy sets and interval valued intuitionistic fuzzy sets. International Journal of Fuzzy Systems, 21(8), 2327-2339.

Zadeh, L. A. (1965). Fuzzy sets. Information and Control, 8(3), 338-353. 